Also available at http://amc.imfm.si

ISSN 1855-3966 (printed edn.), ISSN 1855-3974 (electronic edn.)

ARS MATHEMATICA CONTEMPORANEA 6 (2013) 171-185

\title{
Sharp spectral inequalities for connected bipartite graphs with maximal $Q$-index
}

\author{
Milica Anđelić * \\ Department of Mathematics, University of Aveiro, 3810-193 Aveiro, Portugal \\ Faculty of Mathematics, University of Belgrade, 11000 Belgrade, Serbia \\ C. M. da Fonseca ${ }^{\dagger}$
}

Department of Mathematics, University of Coimbra, 3001-501 Coimbra, Portugal

Tamara Koledin

Faculty of Electrical Engineering, University of Belgrade, 11000 Belgrade, Serbia

\section{Zoran Stanić $\ddagger$}

Faculty of Mathematics, University of Belgrade, 11000 Belgrade, Serbia

Received 31 October 2011, accepted 26 December 2011, published online 11 July 2012

\begin{abstract}
The $Q$-index of a simple graph is the largest eigenvalue of its signless Laplacian. As for the adjacency spectrum, we will show that in the set of connected bipartite graphs with fixed order and size, the bipartite graphs with maximal $Q$-index are the double nested graphs. We provide a sequence of (in)equalities regarding the principal eigenvector of the signless Laplacian of double nested graphs and apply these results to obtain some lower and upper bounds for their $Q$-index. In the end, we give some computational results in order to compare these bounds.
\end{abstract}

Keywords: Double nested graph, signless Laplacian, largest eigenvalue, spectral inequalities.

Math. Subj. Class.: 05C50

* Research supported by CIDMA - Center for Research and Development in Mathematics and Applications, FCT - Fundação para a Ciência e a Tecnologia, through European program COMPETE/FEDER and Serbian Ministry of Education and Science, Project 174033.

${ }^{\dagger}$ Research supported by CMUC - Centro de Matemática da Universidade de Coimbra and FCT - Fundação para a Ciência e a Tecnologia, through European program COMPETE/FEDER

${ }^{\ddagger}$ Research supported by Serbian Ministry of Education and Science, Projects 174012 and 174033.

E-mail addresses: milica.andelic@ua.pt (Milica Anđelić), cmf@mat.uc.pt (C. M. da Fonseca), tamara@etf.rs (Tamara Koledin), zstanic@math.rs (Zoran Stanić) 


\section{Introduction}

Let $G=(V, E)$ be a (simple) graph, of order $\nu=|V|$ and size $\epsilon=|E|$. The signless Laplacian of $G$ is defined to be the matrix $Q=A+D$, where $A(=A(G))$ is the adjacency matrix of $G$, while $D(=D(G))$ is the diagonal matrix of its vertex degrees. The largest eigenvalue (or spectral radius) of $Q$ is usually called the $Q$-index of $G$, and is denoted by $\kappa(=\kappa(G))$. Much interest has been paid recently to this very important spectral invariant. Let us recall that

$$
\Delta+1 \leqslant \kappa \leqslant 2(\nu-1)
$$

where $\Delta$ denotes the maximal vertex degree of the graph, with equality for stars, on the lower bound, and complete graphs, on upper bound [7].

In 2007, Cvetković, Rowlinson, and Simić [6] conjectured that

$$
\kappa \leqslant \nu-1+\bar{d}
$$

where $\bar{d}$ is the average (vertex) degree of a graph. Later Feng and Yu [9] proved that (1.2) is true (cf. also [1]). Many other bounds on $Q$-index for arbitrary (connected) graphs can be found in [8].

We will now describe in brief the structure of a connected double nested graph (or DNG for short). It was first considered in [3, 4] and, independently, under the name of chain graph, in [5], in studying graphs whose least eigenvalue is minimal among the connected (bipartite) graphs of fixed order and size. The vertex set of any such graph $G$ consists of two colour classes (or co-cliques). To specify the nesting, both of them are partitioned into $h$ non-empty cells $\bigcup_{i=1}^{h} U_{i}$ and $\bigcup_{i=1}^{h} V_{i}$, respectively; all vertices in $U_{s}$ are joined (by cross edges) to all vertices in $\bigcup_{k=1} V_{k}$, for $s=1,2, \ldots, h$. Denote by $N_{G}(w)$ the set of neighbors of a vertex $w$. Hence, if $u^{\prime} \in U_{s+1}, u^{\prime \prime} \in U_{s}, v^{\prime} \in V_{t+1}$, and $v^{\prime \prime} \in V_{t}$, then $N_{G}\left(u^{\prime}\right) \subset N_{G}\left(u^{\prime \prime}\right)$ and $N_{G}\left(v^{\prime}\right) \subset N_{G}\left(v^{\prime \prime}\right)$. This claim makes precise the double nesting property. Observe that $1 \leqslant s, t \leqslant h$.

If $m_{s}=\left|U_{s}\right|$ and $n_{s}=\left|V_{s}\right|$, with $s=1,2, \ldots, h$, then $G$ is denoted by

$$
D N G\left(m_{1}, m_{2}, \ldots, m_{h} ; n_{1}, n_{2}, \ldots, n_{h}\right) .
$$

Note that $G$ is connected whenever $m_{1}, n_{1}>0$. Additionally, if some of the remaining parameters are equal to zero, we again get a DNG with a smaller value of $h$. Thus, throughout we assume that all these parameters are greater than zero.

We now introduce some notation to be used later on. Let

$$
M_{s}=\sum_{i=1}^{s} m_{i} \quad \text { and } \quad N_{t}=\sum_{j=1}^{t} n_{j}, \quad \text { for } 1 \leqslant s, t \leqslant h .
$$

Thus $G$ is of order $\nu=M_{h}+N_{h}$ and size $\epsilon=\sum_{s=1}^{h} m_{s} N_{h+1-s}$. Observe that $N_{h+1-s}$ is the degree of a vertex $u \in U_{s}$; the degree of a vertex $v \in V_{t}$ is equal to $M_{h+1-t}$. We also set $M_{s, t}=M_{t}-M_{s-1}$ and, additionally, $M_{1, t}=M_{t}$. 


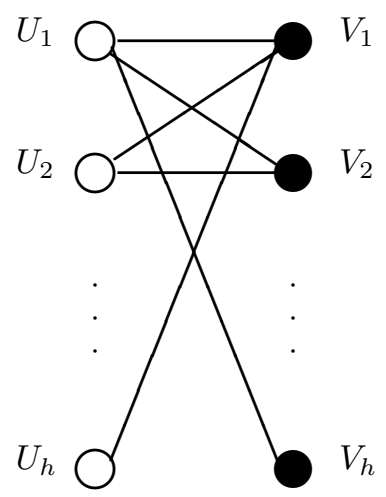

Figure 1: The structure of a double nested graph.

\section{Extremal bipartite graphs}

Let $G$ be a bipartite graph with colour classes $U$ and $V$. First, we state the main result of this section.

Theorem 2.1. If $G$ is a graph for which $\kappa(G)$ is maximal among all connected bipartite graphs of order $\nu$ and size $\epsilon$, then $G$ is a DNG with all pendant edges attached to a common vertex.

Theorem 2.1 means that double nested graphs play the same role among bipartite graphs (with respect to the signless Laplacian index) as nested split graphs among non-bipartite graphs. The same classes of graphs appear as extremal with respect to the adjacency spectra as well, i.e., in the class of all connected (resp. all connected bipartite) graphs of fixed order and size, those with maximal radius with respect to the adjacency matrix are NSGs (resp. DNGs). The proof of Theorem 2.1 is based on the following lemmas, the first of which is taken from [6]. Recall that there exists a unique unit eigenvector corresponding to $\kappa(G)$ having only positive entries; this eigenvector is called the principal eigenvector of $G$.

Lemma 2.2. Let $G^{\prime}$ be the graph obtained from a connected graph $G$ by rotating the edge rs around $r$ to the non-edge position rt. Let $\mathbf{x}=\left(x_{1}, x_{2}, \ldots, x_{\nu}\right)^{T}$ be the principal eigenvector of $G$. If $x_{t} \geqslant x_{s}$ then $\kappa\left(G^{\prime}\right)>\kappa(G)$.

The next lemma will be very helpful when we find a bridge in a graph whose index is assumed to be maximal. Given two rooted graphs $P(=P u)$ and $Q(=Q v)$ with $u$ and $v$ as roots, let $G$ be the graph obtained from the disjoint union $P \dot{\cup} Q$ by adding the edge $u v$. Let $G^{\prime}$ be the graph obtained from the coalescence of $P u$ and $Q v$ by attaching a pendant edge at the vertex identified with $u$ and $v$.

Lemma 2.3. With the above notation, if $P$ and $Q$ are two non-trivial connected graphs then $\kappa(G)<\kappa\left(G^{\prime}\right)$.

Proof. Let $\left(x_{1}, x_{2}, \ldots, x_{\nu}\right)^{T}$ be the principal eigenvector of $G$. Without loss of generality, we may suppose that $x_{u} \leqslant x_{v}$. Let $N_{P}(u)$ be the neigbourhood of $u$ in $P$; since $P$ is 
non-trivial, $N_{P}(u) \neq \emptyset$. Now $G^{\prime}$ is obtained from $G$ by replacing the edges $u w$, with $w \in$ $N_{P}(u)$ by the edges $v w$ and, therefore, $\kappa(G)<\kappa\left(G^{\prime}\right)$, by Lemma 2.2, as required.

In what follows we assume that $G$ has maximal index among the connected bipartite graphs of fixed order and size.

Lemma 2.4. Let $G$ be a graph satisfying the above assumptions, and let $\mathbf{x}=\left(x_{1}, \ldots, x_{\nu}\right)^{T}$ be the principal eigenvector of $G$. If $v$ and $w$ are vertices in the same colour class such that $x_{v} \geqslant x_{w}$, then $\operatorname{deg}(v) \geqslant \operatorname{deg}(w)$.

Proof. Let $U, V$ be the colour classes of $G$. Assuming that $v$ and $w$ are vertices in $V$ such that $x_{v} \geqslant x_{w}$ and $\operatorname{deg}(v)<\operatorname{deg}(w)$, then $\operatorname{deg}(w)>1$ and there exists $u \in U$ such that $v \not u \sim w$. By Lemma 2.3, we may rotate $u w$ to $u v$ to obtain a graph $G^{\prime}$ such that $\kappa\left(G^{\prime}\right)>\kappa(G)$. If $u w$ is a bridge, then $\operatorname{deg}(u)=1$ and, again by Lemma 2.3, $G^{\prime}$ is necessarily connected; but now the maximality of $\kappa(G)$ is contradicted and the proof is complete.

From now on we take the colour classes to be $U=\left\{u_{1}, u_{2}, \ldots, u_{m}\right\}$ and $V=$ $\left\{v_{1}, v_{2}, \ldots, v_{n}\right\}$, with $x_{u_{1}} \geqslant x_{u_{2}} \geqslant \cdots \geqslant x_{u_{m}}$ and $x_{v_{1}} \geqslant x_{v_{2}} \geqslant \cdots \geqslant x_{v_{n}}$. By Lemma 2.4, this ordering coincides with the ordering by degrees in each colour class. In the next lemma we note some consequences of those facts.

Lemma 2.5. Let $G$ be a graph satisfying the above assumptions including those on vertex ordering. Then

(i) the vertices $u_{1}$ and $v_{1}$ are adjacent;

(ii) $u_{1}$ is adjacent to every vertex in $V$, and $v_{1}$ is adjacent to every vertex in $U$;

(iii) if the vertex $u$ is adjacent to $v_{k}$, then $u$ is adjacent to $v_{j}$, for all $j<k$, and if the vertex $v$ is adjacent to $u_{k}$, then $v$ is adjacent to $u_{j}$, for all $j<k$.

Proof. First we consider bridges in G. By Lemma 2.3, all bridges are pendant edges. By Lemma 2.2, all pendant edges are attached at the same vertex, and this vertex $w$ is such that $x_{w}$ is maximal. Without loss of generality, $x_{u_{1}} \geqslant x_{v_{1}}$ and $w=u_{1}$. It follows that the result holds if $G$ is a tree and, consequently, $G$ is a star. Accordingly, we suppose that $G$ is not a tree.

To prove (i), suppose by way of contradiction that $u_{1} \not v_{1}$. Then $v_{1}$ is adjacent to some vertex $u \in U$, and $u v_{1}$ is not a bridge. By Lemma 2.2, we may rotate $v_{1} u$ to $v_{1} u_{1}$ to obtain a connected bipartite graph $G^{\prime}$ such that $\kappa\left(G^{\prime}\right)>\kappa(G)$, contradicting the maximality of $\kappa(G)$.

To prove (ii), suppose that $u$ is a vertex of $U$ not adjacent to $v_{1}$. Then $u \neq u_{1}$ by (i), $u v$ is not a bridge, and $u$ is adjacent to some vertex $v$ in $V$ other than $v_{1}$. Now we can rotate $u v$ to $u v_{1}$ to obtain a contradiction as before. Secondly, suppose that $v$ is a vertex of $V$ not adjacent to $u_{1}$. Then $v \neq v_{1}$ by (i), again $v u_{1}$ is not a bridge, and a rotation about $v$ yields a contradiction.

To prove (iii), suppose that $u \in U, u \sim v_{k}$ and $u \not v_{j}$ for some $j<k$. Now $u \neq u_{1}$ by (ii), and so $u v_{k}$ is not a bridge. Then we can rotate $u v_{k}$ to $u v_{j}$ to obtain a contradiction. Finally, suppose that $v \in V, v \sim v_{k}$ and $v \not u_{j}$ for some $j<k$. In this case, $v u_{k}$ is not a bridge because $k>1$, and the rotation of $v u_{k}$ to $v u_{j}$ yields a contradiction.

The proof is now finished. 
Taking into account Lemma 2.5 and the definition of a DNG the first part of Theorem 2.1 follows. It remains only to prove that all cut-edges in the observed DNG are pendant edges attached to a common vertex. This easily comes from Lemma 2.3.

\section{$3 Q$-eigenvectors of DNGs}

Here we consider the principal eigenvector of the signless Laplacian of DNGs. In this section (and in the next one, if not told otherwise) we will assume that

$$
\mathbf{x}=\left(x_{1}, \ldots, x_{\nu}\right)^{T}
$$

is a $Q$-eigenvector of $G$ with all positive entries, which is usually normalized, i.e.,

$$
\sum_{i=1}^{\nu} x_{i}=1
$$

The entries of $\mathbf{x}$ are also called the weights of the corresponding vertices. We first observe that all vertices within the sets $U_{s}$ or $V_{t}$, for $1 \leqslant s, t \leqslant h$, have the same weights, since they belong to the same orbit of $G$. Let $x_{u}=a_{s}$, if $u \in U_{s}$, while $x_{v}=b_{t}$, if $v \in V_{t}$.

From the eigenvalue equations for $\kappa$, applied to any vertex from $U_{s}$ or $V_{t}$, we get

$$
\kappa a_{s}=N_{h+1-s} a_{s}+\sum_{j=1}^{h+1-s} n_{j} b_{j}, \quad \text { for } s=1, \ldots, h,
$$

and

$$
\kappa b_{t}=M_{h+1-t} b_{t}+\sum_{i=1}^{h+1-t} m_{i} a_{i}, \quad \text { for } t=1, \ldots, h .
$$

By normalization we have

$$
\sum_{i=1}^{h} m_{i} a_{i}+\sum_{j=1}^{h} n_{j} b_{j}=1
$$

and, from (3.1), we easily get

$$
a_{s}=\frac{1}{\kappa-N_{h+1-s}} \sum_{j=1}^{h+1-s} n_{j} b_{j}, \quad \text { for } s=1, \ldots, h .
$$

From (3.2) we have

$$
b_{t}=\frac{1}{\kappa-M_{h+1-t}} \sum_{i=1}^{h+1-t} m_{i} a_{i}, \quad \text { for } t=1, \ldots, h,
$$

and therefore, using (3.3), we have

$$
a_{s}=\frac{1}{\kappa-N_{h+1-s}}\left(1-\sum_{i=1}^{h} m_{i} a_{i}-\sum_{j=h+2-s}^{h} n_{j} b_{j}\right), \quad \text { for } s=1, \ldots, h,
$$


or, using (3.2) for $t=1$,

$$
a_{s}=\frac{1}{\kappa-N_{h+1-s}}\left(1-\left(\kappa-M_{h}\right) b_{1}-\sum_{j=h+2-s}^{h} n_{j} b_{j}\right), \quad \text { for } s=1, \ldots, h .
$$

Similarly,

$$
b_{t}=\frac{1}{\kappa-M_{h+1-t}}\left(1-\left(\kappa-N_{h}\right) a_{1}-\sum_{i=h+2-t}^{h} m_{i} a_{i}\right), \quad \text { for } t=1, \ldots, h .
$$

Setting $a_{h+1}=b_{h+1}=0$ and $N_{0}=0$, from (3.4) and (3.6), together with (3.3), we get successively

$$
\left(\kappa-N_{h-s}\right) a_{s+1}-\left(\kappa-N_{h+1-s}\right) a_{s}=-n_{h+1-s} b_{h+1-s}, \quad \text { for } s=1, \ldots, h-1,
$$

and

$$
\left(\kappa-n_{1}\right) a_{h}=n_{1} b_{1}, \quad \text { for } s=h .
$$

Since all components of $\mathbf{x}$ are positive and $\kappa \geqslant \Delta+1(1.1)$, it comes

$$
a_{s+1} \leqslant a_{s}, \quad \text { for } s=1, \ldots, h-1,
$$

and

$$
b_{t+1} \leqslant b_{t}, \quad \text { for } t=1, \ldots, h-1 .
$$

Furthermore, by setting $s=h$ in (3.2), we obtain

$$
\left(\kappa-m_{1}\right) b_{h}=m_{1} a_{1} .
$$

Moreover, substituting $s=1$ in (3.1) and $t=1$ in (3.2) and applying in (3.3) we get

$$
\left(\kappa-N_{h}\right) a_{1}+\left(\kappa-M_{h}\right) b_{1}=1,
$$

and finally

$$
a_{s}=\frac{1}{\kappa-N_{h+1-s}}\left(\left(\kappa-N_{h}\right) a_{1}-\sum_{j=h+2-s}^{h} n_{j} b_{j}\right) .
$$

Next we focus our attention on bounding $a_{i}$ 's and $b_{j}$ 's.

Lemma 3.1. For any $s=1, \ldots, h$, we have

$$
\frac{N_{h+1-s} b_{h+1-s}}{\kappa-N_{h+1-s}} \leqslant a_{s} \leqslant \frac{N_{h+1-s} b_{1}}{\kappa-N_{h+1-s}} .
$$

Proof. From (3.4), we have

$$
a_{s}=\frac{1}{\kappa-N_{h+1-s}} \sum_{j=1}^{h+1-s} n_{j} b_{j}
$$

Therefore, (3.11) immediately follows since $b_{j}$ 's are strictly decreasing, from (3.8). 
Lemma 3.2. For any $s=1, \ldots, h$,

$$
a_{s} \leqslant a_{1}\left(1-\frac{N_{h+2-s, h}}{\kappa-N_{h+1-s}}\left(1+\frac{m_{1}}{\kappa-m_{1}}\right)\right),
$$

Proof. The inequality (3.12) follows from (3.10), since $b_{i}$ 's are strictly decreasing, bearing in mind (3.9) as well.

Lemma 3.3. For any $s=1, \ldots, h$,

$$
a_{s} \geqslant \frac{a_{1}}{\kappa-N_{h+1-s}}\left(1-\sum_{i=1}^{s-1} \frac{n_{h+1-i} M_{i}}{\kappa-M_{i}}\right) .
$$

Proof. By induction on $s$. For $s=1$, the inequality holds trivially. Assume next that

$$
a_{s} \geqslant \frac{a_{1}}{\kappa-N_{h+1-s}}\left(1-\sum_{i=1}^{s-1} \frac{n_{h+1-i} M_{i}}{\kappa-M_{i}}\right),
$$

for $s>1$. Then

$$
\begin{aligned}
a_{s+1} & =\frac{1}{\kappa-N_{h-s}} \sum_{j=1}^{h-s} n_{j} b_{j} \\
& =\frac{1}{\kappa-N_{h-s}}\left(\left(\kappa-N_{h+1-s}\right) a_{s}-N_{h+1-s} b_{h+1-s}\right) \\
& \geqslant \frac{a_{1}}{\kappa-N_{h-s}}\left(1-\sum_{i=1}^{s-1} \frac{n_{h+1-i} M_{i}}{\kappa-M_{i}}\right)-\frac{N_{h+1-s} M_{s} a_{1}}{\left(\kappa-N_{h-s}\right)\left(\kappa-M_{s}\right)} \\
& =\frac{a_{1}}{\kappa-N_{h-s}}\left(1-\sum_{i=1}^{s} \frac{n_{h+1-i} M_{i}}{\kappa-M_{i}}\right) .
\end{aligned}
$$

This ends the proof.

Lemma 3.4. For any $s=1, \ldots, h$, we have

$$
a_{s} \leqslant \frac{b_{1}}{\kappa-N_{h+1-s}}\left(N_{h+1-s}-\frac{\kappa f_{h+1-s}}{\left(\kappa-n_{1}\right)\left(\kappa-M_{s}\right)}\right),
$$

where

$$
f_{h+1-s}=\sum_{j=1}^{h+1-s} n_{j} M_{h+2-j, h} .
$$

Proof. From (3.4) and (3.12) applied to $b_{j}$, we get

$$
\begin{aligned}
a_{s} & =\frac{1}{\kappa-N_{h+1-s}} \sum_{j=1}^{h+1-s} n_{j} b_{j} \\
& \leqslant \frac{1}{\kappa-N_{h+1-s}} \sum_{j=1}^{h+1-s} n_{j} b_{1}\left(1-\frac{M_{h+2-j, h}}{\kappa-M_{h+1-j}}\left(1+\frac{n_{1}}{\kappa-n_{1}}\right)\right) \\
& \leqslant \frac{b_{1}}{\kappa-N_{h+1-s}}\left(N_{h+1-s}-\frac{\kappa f_{h+1-s}}{\left(\kappa-n_{1}\right)\left(\kappa-M_{s}\right)}\right) .
\end{aligned}
$$

The proof is now complete. 


\section{Some bounds on the $Q$-index of a DNG}

In this section we obtain some upper and lower bounds on the $Q$-index of DNGs using the eigenvalue and the matrix technique. We also emphasize that our main goal is to consider the estimation of the $Q$-index of large graphs. Before we proceed, we provide the following observations.

First, if $h=1$ we get a complete bipartite graph $K_{m_{1}, n_{1}}$, whose $Q$-index is equal to $m_{1}+n_{1}=\nu$ [2]. Furthermore, since the $Q$-index of an arbitrary graph increases by inserting edges (cf. [6]), we have

$$
\kappa \leqslant \nu
$$

for any (not necessarily connected) DNG.

Otherwise, if $h>1$ is fixed but the graph size is not, using the same previous arguments, the maximal $Q$-index would appear in $D N G\left(m_{1}, 1, \ldots, 1 ; n_{1}, 1, \ldots, 1\right)$. The computational results suggest this will happen when $\left|m_{1}-n_{1}\right| \leqslant 1$. So, these cases are not interesting for our research and, therefore, we will assume that $h>1$ and the size is fixed.

\subsection{Eigenvalue technique}

Now we establish some bounds for the $Q$-index of DNGs using the eigenvalue technique. We start with lower bounds.

Proposition 4.1. If $G$ is a connected $D N G$, then

$$
\kappa \geqslant \max _{1 \leqslant k \leqslant h}\left\{M_{h+1-k}+N_{k}\right\}
$$

Proof. On the one hand, from (3.2), we get

$$
b_{k}=\frac{1}{\kappa-M_{h+1-k}} \sum_{i=1}^{h+1-k} m_{i} a_{i} \geqslant \frac{M_{h+1-k} a_{h+1-k}}{\kappa-M_{h+1-k}},
$$

since, from (3.7), $a_{i}$ 's are decreasing. On the other hand, from (3.1), we get

$$
a_{h+1-k}=\frac{1}{\kappa-N_{k}} \sum_{j=1}^{k} n_{j} b_{j} \geqslant \frac{N_{k} b_{k}}{\kappa-N_{k}},
$$

since $b_{j}$ 's are decreasing, from (3.8). From the last two inequalities we get

$$
\kappa\left(\kappa-\left(M_{h+1-k}+N_{k}\right)\right) \geqslant 0,
$$

which is equivalent to

$$
\kappa \geqslant M_{h+1-k}+N_{k}
$$

In particular, for $k=h$ and $k=1$, we obtain the following corollary.

Corollary 4.2. If $G$ is a connected $D N G$, then

$$
\kappa \geqslant m_{1}+N_{h} \text { and } \kappa \geqslant n_{1}+M_{h} .
$$


Proposition 4.3. If $G$ is a connected $D N G$, then

$$
\kappa \geqslant \frac{1}{2}\left(t+\frac{\epsilon}{N_{h}}+\sqrt{\left(t-\frac{\epsilon}{N_{h}}\right)^{2}+4 \hat{e}_{h}^{*}}\right)
$$

where

$$
t=\frac{\sum_{i=1}^{h} m_{i} N_{h+1-i}^{3}}{\sum_{i=1}^{h} m_{i} N_{h+1-i}^{2}} \text { and } \hat{e}_{h}^{*}=\sum_{i=1}^{h} m_{i} \frac{N_{h+1-i}^{2}}{N_{h}} .
$$

Proof. Let $\mathbf{y}=\left(y_{1}, \ldots, y_{\nu}\right)^{T}$ be a vector whose components are indexed by the vertices of $G$, and let $y_{u}=N_{h+1-i}$ if $u \in U_{i}$, for some $i \in\{1, \ldots, h\}$, or, otherwise, $y_{v}=q=\kappa-t$, for some $t$, if $v \in V_{j}$ for some $j \in\{1, \ldots, h\}$. Substituting $y$ into the Rayleigh quotient (see, e.g., [8, p. 49]) we obtain

$$
\kappa \geqslant \frac{2 \sum_{i=1}^{h} m_{i} N_{h-1+i}^{2} q+\sum_{i=1}^{h} m_{i} N_{h+1-i}^{3}+\sum_{i=1}^{h} n_{i} M_{h-1+i} q^{2}}{\sum_{i=1}^{h} m_{i} N_{h+1-i}^{2}+N_{h} q^{2}}
$$

due to Rayleigh's principle which reads $\frac{y^{T} Q y}{y^{T} y} \leqslant \kappa$. Since $q=\kappa-t$, we get

$$
N_{h} q^{3}+\left(N_{h} t-\epsilon\right) q^{2}-\sum_{i=1}^{h} m_{i} N_{h+1-i}^{2} q \geqslant \sum_{i=1}^{h} m_{i} N_{h+1-i}^{3}-t \sum_{i=1}^{h} m_{i} N_{h+1-i}^{2} .
$$

Choosing

$$
t=\frac{\sum_{i=1}^{h} m_{i} N_{h+1-i}^{3}}{\sum_{i=1}^{h} m_{i} N_{h+1-i}^{2}}
$$

and having in mind that $N_{1} \leqslant t \leqslant N_{h}$, we immediately get a quadratic inequality in $q$ and the proof is concluded.

Proposition 4.4. If $G$ is a connected $D N G$, then

$$
\kappa \leqslant \frac{1}{2}\left(\nu+\sqrt{\nu^{2}-4\left(M_{h} N_{h}-\epsilon\right)}\right) .
$$

Proof. From (3.1), with $s=h$, and from (3.3), recalling (3.11), we get

$$
\left(\kappa-M_{h}\right) b_{1}=\sum_{i=1}^{h} m_{i} a_{i} \leqslant \sum_{i=1}^{h} m_{i} \frac{N_{h+1-i}}{\kappa-N_{h+1-s}} b_{1} .
$$

Then, we obtain

$$
\left(\kappa-M_{h}\right)\left(\kappa-N_{h}\right) \leqslant \epsilon,
$$

and, therefore, from the quadratic inequality

$$
\kappa^{2}-\left(M_{h}+N_{h}\right) \kappa+M_{h} N_{h}-\epsilon \leqslant 0,
$$

we obtain $\kappa_{1} \leqslant \kappa \leqslant \kappa_{2}$ where $\kappa_{1}$ and $\kappa_{2}$ are the solutions of the associated quadratic equality, and this completes the proof. 
The next two bounds improve the bound (4.2). We recall that $f_{h+1-i}$ is defined in Lemma 3.4.

Proposition 4.5. If $G$ is a connected $D N G$, then

$$
\kappa \leqslant \frac{1}{2}\left(\nu+\sqrt{\nu^{2}-4\left(M_{h} N_{h}-\epsilon^{\prime}\right)}\right)
$$

where

$$
\epsilon^{\prime}=\epsilon-\frac{\nu\left(\nu-N_{h}\right)}{\left(\nu-n_{1}\right)^{2}\left(\nu-m_{1}\right)} \sum_{i=1}^{h} m_{i} f_{h+1-i} .
$$

Proof. As in the proof of Proposition 4.4, we have

$$
\left(\kappa-M_{h}\right) b_{1}=\sum_{i=1}^{h} m_{i} a_{i} .
$$

Using (3.12), we get

$$
\kappa-M_{h} \leqslant \sum_{i=1}^{h} \frac{m_{i}}{\kappa-N_{h+1-i}}\left(N_{h+1-i}-\frac{\kappa f_{h+1-i}}{\left(\kappa-n_{1}\right)\left(\kappa-M_{i}\right)}\right),
$$

and therefore

$$
\left(\kappa-M_{h}\right)\left(\kappa-N_{h}\right) \leqslant \epsilon-\frac{\kappa\left(\kappa-N_{h}\right)}{\left(\kappa-n_{1}\right)^{2}\left(\kappa-m_{1}\right)} \sum_{i=1}^{h} m_{i} f_{h+1-i} .
$$

Taking into account that $\kappa \leqslant \nu$, from Proposition 4.4 it follows

$$
\left(\kappa-M_{h}\right)\left(\kappa-N_{h}\right) \leqslant \epsilon^{\prime}
$$

and the proof ends.

The next result may be proved in a similar way.

Proposition 4.6. If $G$ is a connected $D N G$, then

$$
\kappa \leqslant \frac{1}{2}\left(\nu+\sqrt{\nu^{2}-4\left(M_{h} N_{h}-\epsilon^{\prime \prime}\right)}\right)
$$

where

$$
\epsilon^{\prime \prime}=\epsilon-\frac{\kappa^{\prime}\left(\kappa^{\prime}-N_{h}\right)}{\left(\kappa^{\prime}-n_{1}\right)^{2}\left(\kappa^{\prime}-m_{1}\right)} \sum_{i=1}^{h} m_{i} f_{h+1-i}
$$

for

$$
\kappa^{\prime}=\frac{1}{2}\left(\nu+\sqrt{\nu^{2}-4\left(M_{h} N_{h}-\epsilon^{\prime}\right)}\right) .
$$




\subsection{Matrix technique}

The partition

$$
V=\bigcup_{k=1}^{h} U_{k} \cup \bigcup_{k=1}^{h} V_{k}
$$

is equitable since every vertex in $U_{i}$ and every vertex in $V_{i}$ have the same number of neighbors in $U_{j}$ and $V_{j}$, for all $i, j \in\{1,2, \ldots, h\}$. Let $A_{D}$ be the signless Laplacian divisor matrix of a $D N G\left(m_{1}, \ldots, m_{h} ; n_{1}, \ldots, n_{h}\right)$ with respect to the equitable partition (4.3). The matrix $A_{D}$ has the following form:

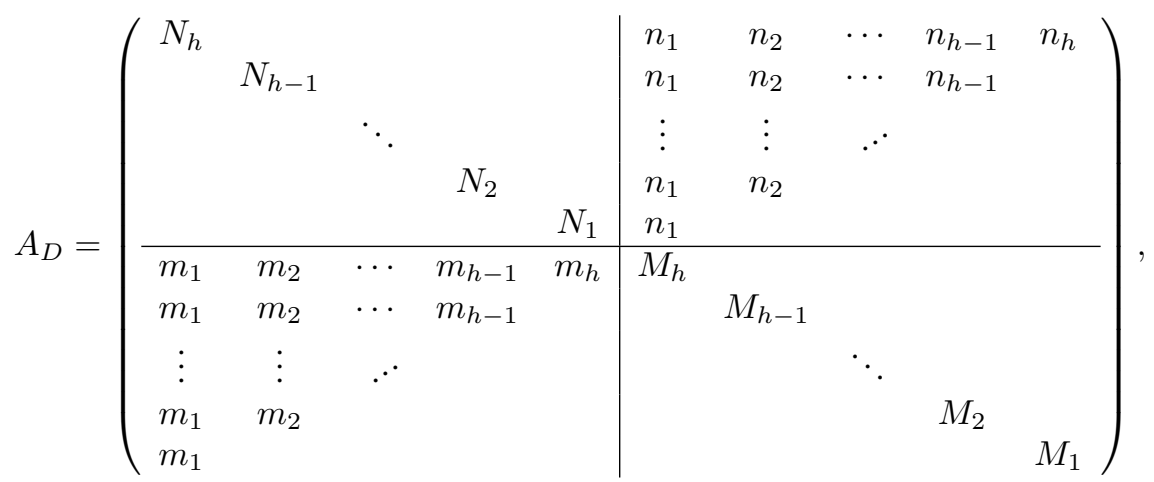

where the non-mentioned entries are to be read as zero. Setting

$$
N=\left(\begin{array}{rrrrr}
n_{1} & n_{2} & \cdots & n_{h-1} & n_{h} \\
n_{1} & n_{2} & \cdots & n_{h-1} & \\
\vdots & \vdots & . . & & \\
n_{1} & n_{2} & & & \\
n_{1} & & & &
\end{array}\right), \quad M=\left(\begin{array}{rrrrr}
m_{1} & m_{2} & \cdots & m_{h-1} & m_{h} \\
m_{1} & m_{2} & \cdots & m_{h-1} & \\
\vdots & \vdots & . \cdot & & \\
m_{1} & m_{2} & & & \\
m_{1} & & & &
\end{array}\right) \text {, }
$$

$D_{1}=\operatorname{diag}\left(N_{h}, \ldots, N_{1}\right)$, and $D_{2}=\operatorname{diag}\left(M_{h}, \ldots, M_{1}\right), A_{D}$ can be rewritten in the compact block form

$$
A_{D}=\left(\begin{array}{cc}
D_{1} & N \\
M & D_{2}
\end{array}\right) .
$$

In order to obtain more bounds we set

$$
P=\left(\begin{array}{cc}
0 & x I \\
I & 0
\end{array}\right)
$$

for some $x \neq 0$. Since the matrices

$$
P A_{D} P^{-1}=\left(\begin{array}{cc}
D_{2} & x M \\
x^{-1} N & D_{1}
\end{array}\right)
$$

and $A_{D}$ are similar, they have the same index. We choose $x$ such that the sum in the first row and the $(h+1)$-th row are equal. It leads to $M_{h} x^{2}-\left(N_{h}-M_{h}\right) x-N_{h}=0$, i.e., $x=\frac{N_{h}}{M_{h}}$. By Frobenius Theorem [11, Theorem 3.1.1], we have

$$
\min _{1 \leqslant i \leqslant n} R_{i} \leqslant \kappa \leqslant \max _{1 \leqslant i \leqslant n} R_{i}
$$


where $R_{i}$ stands for the sum of elements in the $i$-th row of $P A_{D} P^{-1}$. Using this, we get the following result.

Proposition 4.7. If $G$ is a connected $D N G$, then

$$
\min \left\{n_{1}\left(\frac{N_{h}}{M_{h}}+1\right), n_{1}\left(\frac{M_{h}}{N_{h}}+1\right)\right\} \leqslant \kappa \leqslant N_{h}+M_{h}=\nu .
$$

Clearly, the upper bound does not provide a decisive progress in our quest (recall (4.1)). We will establish some more interesting improvements next.

Let $R_{i}$ be the sum of the entries in row $i$ of the matrix $A_{D}$. It is easy to confirm that

$$
\begin{aligned}
R_{i} & =2 N_{h-i+1}, \quad \text { for } i \in\{1, \ldots, h\} \\
& =2 M_{h-i+1}, \quad \text { for } i \in\{h+1, \ldots, 2 h\},
\end{aligned}
$$

and, therefore,

$$
\max R_{i}=\max \left\{2 N_{h}, 2 M_{h}\right\}
$$

By Frobenius Theorem

$$
\min \left\{2 n_{1}, 2 m_{1}\right\} \leqslant \kappa \leqslant \max \left\{2 N_{h}, 2 M_{h}\right\} .
$$

Here the upper bound does not make any (general) improvement since $\max \left\{2 N_{h}, 2 M_{h}\right\} \geqslant$ $\nu$ (compare (4.1)), so next we use the result of Minc (see [10]), which for the matrix $A_{D}$ reads:

$$
\min _{i} \frac{\sum_{j=1}^{h}\left(A_{D}\right)_{i j} R_{j}}{R_{i}} \leqslant \kappa \leqslant \max _{i} \frac{\sum_{j=1}^{h}\left(A_{D}\right)_{i j} R_{j}}{R_{i}} .
$$

Proposition 4.8. If $G$ is a connected $D N G$, then

$$
\min \left\{n_{1}+M_{h}, m_{1}+N_{h}\right\} \leqslant \kappa \leqslant \max \left\{\frac{\epsilon}{N_{h}}+N_{h}, \frac{\epsilon}{M_{h}}+M_{h}\right\} .
$$

The bounds (4.6) obviously improve both (4.4) and (4.5), but the lower bound is still rough comparing with Corollary 4.2.

\section{Computational results}

In this final section, we provide several examples which can help to gain a better insight into the quality of the bounds obtained in the previous section.

We compute the lower bounds of Propositions 4.1 and 4.3, and the upper bounds from Propositions 4.4, 4.5, 4.6, and 4.8. One observes that the bound from Proposition 4.1 is always integral. The number of vertices in the corresponding DNG is also given in every example since it makes another upper bound (cf. (4.1)). It can be easy checked that the lower bounds from Corollary 4.2 and Propositions 4.7 and 4.8, all of them having simple expressions, are rough in some cases, and therefore they are not considered in our examples. We also compute the relative errors in each case.

Example 5.1. First we consider a randomly chosen DNG with small number of vertices and some larger DNGs derived from the previous one:

$$
\begin{aligned}
& G_{1}=D N G(2,2,5,3 ; 2,3,1,1) ; \\
& G_{2}=D N G(10,10,25,15 ; 10,15,5,5) ; \\
& G_{3}=D N G(200,200,500,300 ; 200,300,100,100) ;
\end{aligned}
$$




\begin{tabular}{|c||c|c|c|c|c|c|c|c|}
\hline & Prop. 4.3 & Prop. 4.1 & $\kappa$ & Prop. 4.8 & Prop 4.6 & Prop 4.5 & Prop 4.4 & $\nu$ \\
\hline \hline$G_{1}$ & 13.6785 & 14 & 15.6451 & 16.7500 & 17.0210 & 17.0550 & 17.4530 & 19 \\
& $-12.57 \%$ & $-10.52 \%$ & & $7.06 \%$ & $8.79 \%$ & $9.01 \%$ & $11.56 \%$ & $21.44 \%$ \\
\hline$G_{2}$ & 68.3923 & 70 & 78.2257 & 83.7500 & 85.1052 & 85.2749 & 87.2649 & 95 \\
& $-12.57 \%$ & $-10.52 \%$ & & $7.06 \%$ & $8.79 \%$ & $9.01 \%$ & $11.56 \%$ & $21.44 \%$ \\
\hline$G_{3}$ & 1367.8452 & 1400 & 1564.5133 & 1675.0000 & 1702.1030 & 1705.4985 & 1745.2987 & 1900 \\
& $-12.57 \%$ & $-10.52 \%$ & & $7.06 \%$ & $8.79 \%$ & $9.01 \%$ & $11.56 \%$ & $21.44 \%$ \\
\hline
\end{tabular}

Notice that $\kappa\left(G_{2}\right)$ (resp. $\left.\kappa\left(G_{3}\right)\right)$ is very close to $5 \kappa\left(G_{1}\right)$ (resp. $100 \kappa\left(G_{3}\right)$ ); we get $5 \kappa\left(G_{1}\right)-\kappa\left(G_{2}\right) \approx 10^{-7}$. Since the similar fact holds for all bounds obtained (compare the corresponding propositions), we get the same results for the relative errors.

Example 5.2. Here we consider the DNGs obtained from $G_{1}$ by multiplying some of its parameters:

$$
\begin{aligned}
& H_{1}=D N G(2000,2,5,3 ; 2,3,1,1000) ; \\
& H_{2}=D N G(2000,2,5,3 ; 2,3,1000,1) ; \\
& H_{3}=D N G(2000,2,5,3 ; 2,3000,1,1) ; \\
& H_{4}=D N G(2000,2,5,3 ; 2000,3,1,1) ;
\end{aligned}
$$

\begin{tabular}{|c||c|c|c|c|c|c|c|c|}
\hline & Prop. 4.3 & Prop. 4.1 & $\kappa$ & Prop. 4.8 & Prop 4.6 & Prop 4.5 & Prop 4.4 & $\nu$ \\
\hline \hline$H_{1}$ & 3006.0284 & 3006 & 3006.0287 & 3011.0164 & 3008.2682 & 3008.2960 & 3012.6750 & 3016 \\
& $-8 \cdot 10^{-6} \%$ & $-1 \cdot 10^{-3} \%$ & & $0.17 \%$ & $0.07 \%$ & $0.08 \%$ & $0.22 \%$ & $0.33 \%$ \\
\hline$H_{2}$ & 3008.0175 & 3007 & 3008.0177 & 3012.0104 & 3009.8145 & 3009.8323 & 3013.3388 & 3016 \\
& $-5 \cdot 10^{-6} \%$ & $-0.03 \%$ & & $0.13 \%$ & $0.06 \%$ & $0.06 \%$ & $0.18 \%$ & $0.27 \%$ \\
\hline$H_{3}$ & 5010.9908 & 5009 & 5010.9909 & 5010.9980 & 5011.7199 & 5011.7199 & 5012.2008 & 5014 \\
& $-5 \cdot 10^{-7} \%$ & $-0.04 \%$ & & $1 \cdot 10^{-4} \%$ & $0.15 \%$ & $0.15 \%$ & $0.02 \%$ & $0.06 \%$ \\
\hline$H_{4}$ & 4014.9731 & 4010 & 4014.9732 & 4014.9866 & 4014.9800 & 4014.9800 & 4014.9933 & 4015 \\
& $-3 \cdot 10^{-6} \%$ & $-0.12 \%$ & & $3 \cdot 10^{-4} \%$ & $2 \cdot 10^{-4} \%$ & $2 \cdot 10^{-4} \%$ & $5 \cdot 10^{-4} \%$ & $7 \cdot 10^{-4} \%$ \\
\hline
\end{tabular}

In this example all bounds are (more or less) close to the exact value of $Q$-index. We already pointed that the bounds obtained in Propositions 4.5 and 4.6 are the improvements of the one obtained in Proposition 4.4. This example shows that the bound from Proposition 4.8 is incomparable to them. In opposition to the previous example, here Proposition 4.1 gives a better estimation than Proposition 4.3.

Example 5.3. The parameters of the following DNGs are obtained by multiplying the parameters of $G_{1}$ by $1,10,100$ or 1000 ad hoc.

$$
\begin{aligned}
& I_{1}=D N G(2,2,5,3 ; 2000,300,10,1) ; \\
& I_{2}=D N G(2,2,5,3 ; 2,30,100,1000) ; \\
& I_{3}=D N G(2000,200,50,30 ; 2000,300,10,1) ;
\end{aligned}
$$

\begin{tabular}{|c||c|c|c|c|c|c|c|c|}
\hline & Prop. 4.3 & Prop. 4.1 & $\kappa$ & Prop. 4.8 & Prop 4.6 & Prop 4.5 & Prop 4.4 & $\nu$ \\
\hline \hline$I_{1}$ & 2255.0867 & 2314 & 2316.3632 & 2322.5716 & 2322.5716 & 2322.5733 & 2322.5737 & 2323 \\
& $-2.65 \%$ & $-0.10 \%$ & & $0.26 \%$ & $0.27 \%$ & $0.27 \%$ & $0.27 \%$ & $0.29 \%$ \\
\hline$I_{2}$ & 1118.5026 & 1134 & 1134.0007 & 1134.3799 & 1134.4002 & 1134.4000 & 1134.4002 & 1144 \\
& $-1.37 \%$ & $-6 \cdot 10^{-5} \%$ & & $0.03 \%$ & $0.04 \%$ & $0.04 \%$ & $0.04 \%$ & $0.88 \%$ \\
\hline$I_{3}$ & 4562.6064 & 4550 & 4562.6584 & 4563.2717 & 4563.1367 & 4563.1369 & 4563.6312 & 4591 \\
& $-0.28 \%$ & $-1 \cdot 10^{-3} \%$ & & $0.01 \%$ & $0.01 \%$ & $0.01 \%$ & $0.02 \%$ & $0.62 \%$ \\
\hline
\end{tabular}


Taking into account the lower bound of Proposition 4.3, one can conclude that its deviation from the exact value is expected for $I_{1}$ (and other similar graphs). Note that Proposition 4.6 will often give better bound than Proposition 4.5, but not always - see graphs $I_{2}$ or $J_{2}$ in the next example.

Example 5.4. Finally, we consider the extensions of the original graphs:

$$
\begin{aligned}
& J_{1}=D N G(2,2,5,3,2,3,1,1 ; 2,3,1,1,2,2,5,3) \\
& J_{2}=D N G(20000,2,5,3,10,10,10,10 ; 2,3,1,10000,10,10,10,10) ; \\
& J_{3}=D N G(2,2,5,3,1,1,1,1 ; 2000,300,10,1,1,1,1,1)
\end{aligned}
$$

\begin{tabular}{|c||c|c|c|c|c|c|c|c|}
\hline & Prop. 4.3 & Prop. 4.1 & $\kappa$ & Prop. 4.8 & Prop 4.6 & Prop 4.5 & Prop 4.4 & $\nu$ \\
\hline \hline$J_{1}$ & 23.1888 & 23 & 27.4601 & 29.0526 & 32.3032 & 32.3022 & 32.8203 & 38 \\
& $-15.56 \%$ & $-16.24 \%$ & & $5.80 \%$ & $17.64 \%$ & $17.64 \%$ & $19.52 \%$ & $38.38 \%$ \\
\hline$J_{2}$ & 30065.9176 & 30046 & 30065.9178 & 30080.9446 & 30072.6747 & 30072.7003 & 30085.9668 & 30096 \\
& $-6 \cdot 10^{-7 \%}$ & $-0.07 \%$ & & $0.05 \%$ & $0.02 \%$ & $0.02 \%$ & $0.07 \%$ & $0.10 \%$ \\
\hline$J_{3}$ & 2313.0140 & 2324 & 2327.5409 & 2330.8445 & 2330.8452 & 2330.8452 & 2330.8456 & 2331 \\
& $-0.62 \%$ & $-0.15 \%$ & & $0.14 \%$ & $0.14 \%$ & $0.14 \%$ & $0.14 \%$ & $0.15 \%$ \\
\hline
\end{tabular}

The bounds obtained will be used in a forthcoming research regarding the graphs with maximal $Q$-index and fixed (but high) orders and also a fixed particular size. We also remark that the results could be also compared to the corresponding bounds obtained for the adjacency spectra.

\section{Acknowledgment}

The authors thank two anonymous referees for providing constructive comments in improving the contents of this paper.

\section{References}

[1] M. Anđelić, C. M. da Fonseca, S. K. Simić and D. V. Tošić, Connected graphs of fixed order and size with maximal $Q$-index: Some spectral bounds, Discrete Appl. Math. 160 (2012), 448-459.

[2] M. Anđelić, T. Koledin and Z. Stanić, Nested graphs with bounded second largest (signless Laplacian) eigenvalue, Electron. J. Linear Algebra 24 (2012), to appear.

[3] F. K. Bell, D. Cvetković, P. Rowlinson and S. K. Simić, Graphs for which the least eigenvalue is minimal, I, Linear Algebra Appl. 429 (2008), 234-241.

[4] F. K. Bell, D. Cvetković, P. Rowlinson and S. K. Simić, Graphs for which the least eigenvalue is minimal, II, Linear Algebra Appl. 429 (2008), 2168-2179.

[5] A. Bhattacharya, S. Friedland and U. N. Peled, On the first eigenvalue of bipartite graphs, Electron. J. Combin. 15 (2008), R\#144.

[6] D. Cvetković, P. Rowlinson and S. K. Simić, Eigenvalue bounds for the signless Laplacian, Publ. Inst. Math. (Beograd), 81 (95) (2007), 11-27.

[7] D. Cvetković, P. Rowlinson and S. K. Simić, Signless Laplacians of finite graphs, Linear Algebra Appl. 423 (2007), 155-171.

[8] D. Cvetković, P. Rowlinson and S. K. Simić, An Introduction to the Theory of Graph Spectra, Cambridge University Press, 2009. 
[9] L.-H. Feng, G.-H. Yu, On three conjectures involving the signless Laplacian spectral radius of graphs, Publ. Inst. Math. (Beograd) 85 (99) (2009), 35-38.

[10] S.-L. Liu, Bounds for the greatest characteristic root of a nonnegative matrix, Linear Algebra Appl. 239 (1996), 151-160.

[11] M. Marcus and H. Minc, A Survey of Matrix Theory and Matrix Inequalities, Dover Publications Inc., New York, 1992; unabridged reprint of the corrected 1969 printing, Prindle, Weber, \& Schmidt, Boston. 